\title{
Source brightness fluctuation correction of solar absorption fourier transform mid infrared spectra
}

\author{
T. Ridder, T. Warneke, and J. Notholt \\ Institute of Environmental Physics (IUP), University of Bremen, Otto-Hahn-Allee 1, 28359 Bremen, Germany \\ Received: 10 November 2010 - Published in Atmos. Meas. Tech. Discuss.: 20 January 2011 \\ Revised: 25 May 2011 - Accepted: 27 May 2011 - Published: 9 June 2011
}

\begin{abstract}
The precision and accuracy of trace gas observations using solar absorption Fourier Transform infrared spectrometry depend on the stability of the light source. Fluctuations in the source brightness, however, cannot always be avoided. Current correction schemes, which calculate a corrected interferogram as the ratio of the raw DC interferogram and a smoothed DC interferogram, are applicable only to near infrared measurements. Spectra in the mid infrared spectral region below $2000 \mathrm{~cm}^{-1}$ are generally considered uncorrectable, if they are measured with a MCT detector. Such measurements introduce an unknown offset to MCT interferograms, which prevents the established source brightness fluctuation correction. This problem can be overcome by a determination of the offset using the modulation efficiency of the instrument. With known modulation efficiency the offset can be calculated, and the source brightness correction can be performed on the basis of offset-corrected interferograms.

We present a source brightness fluctuation correction method which performs the smoothing of the raw DC interferogram in the interferogram domain by an application of a running mean instead of high-pass filtering the corresponding spectrum after Fourier transformation of the raw DC interferogram. This smoothing can be performed with the onboard software of commercial instruments.

The improvement of MCT spectra and subsequent ozone profile and total column retrievals is demonstrated. Application to InSb interferograms in the near infrared spectral region proves the equivalence with the established correction scheme.
\end{abstract}

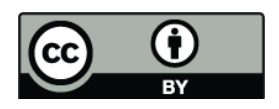

Correspondence to: $\mathrm{T}$. Ridder (tridder@iup.physik.uni-bremen.de)

\section{Introduction}

Ground-based solar absorption Fourier Transform infrared (FTIR) spectrometry (Zander et al., 1983; Goldman et al., 1988; Rinsland et al., 1991) has been established as an accurate and precise method for the detection of trace gases in the atmosphere (Notholt et al., 2003; Velazco et al., 2005). FTIR spectrometry is used by the Network for the Detection of Atmospheric Composition Change (NDACC, 1991) and the Total Carbon Column Observing Network (TCCON, 2005) for the worldwide observation of trace gases.

The accuracy and precision of trace gas concentrations retrieved from FTIR spectra depend on the stability of the light source during the measurement (Beer, 1992; Notholt et al., 1997) since intensity fluctuations can distort the fractional line depth in FTIR spectra (Keppel-Aleks et al., 2007). This distortion of the fractional line depth is due to an additional apodisation which results from the variable source intensity and distorts the instrumental line shape (ILS). Variations in the source brightness caused e.g. by clouds decrease the accuracy and precision, but cannot always be avoided. Thus, one aim of the NDACC and TCCON networks is to reduce the impact of source brightness fluctuations (SBFs) on FTIR spectra by applying a correction to the spectra following the measurements.

The idea of SBF correction was primarily published by Brault (1985) and was recently picked up by Keppel-Aleks et al. (2007). In general, a SBF correction can be applied on Fourier Transform DC interferograms. The measured raw interferogram $I_{\text {raw }}$ is reweighted by the corresponding smoothed interferogram $I_{\text {smooth }}$ in terms of

$I_{\text {corr }}=\frac{I_{\text {raw }}}{I_{\text {smooth }}}$

The reweighting compensates the intensity fluctuations during the measurement and adjusts the modulation height in the interferogram (Fig. 1). 


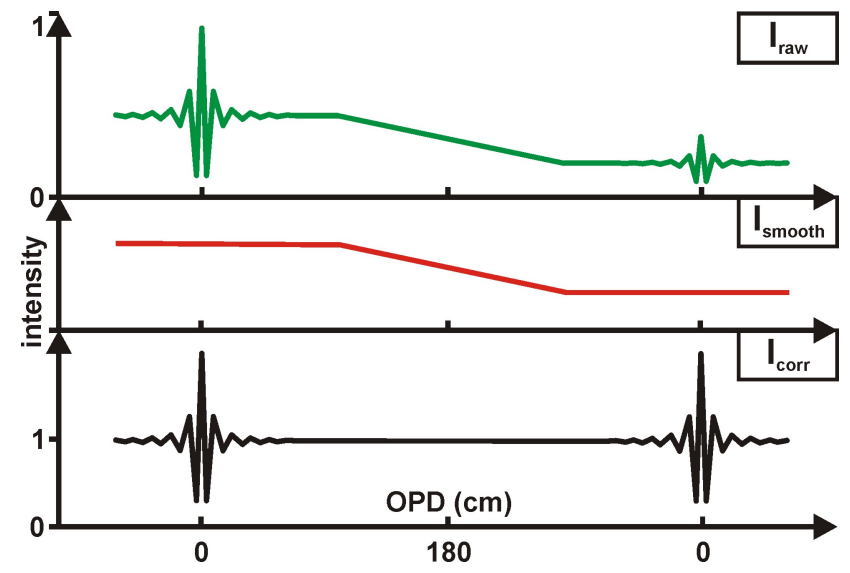

Fig. 1. Schematic of a SBF correction on the basis of one DC interferogram: $I_{\text {raw }}$ (green) demonstrates a raw DC interferogram under the influence of SBF, $I_{\text {smooth }}(\mathrm{red})$ pictures the corresponding smoothed interferogram, and $I_{\text {corr }}$ (black) demonstrates the SBFcorrected interferogram (OPD: optical path difference).

Keppel-Aleks' et al. (2007) analysis is focused on the near infrared spectral region measured simultaneously with an InGaAs diode and a Si diode detector. Their full SBF correction is integrated into the software called "slice-ipp" (developed at the Jet Propulsion Laboratory, Pasadena, CA, USA), and the smoothed interferogram $I_{\text {smooth }}$ is generated by highpass filtering in three steps: (1) taking a fast Fourier Transformation (FFT) of the raw DC interferogram, (2) applying a spectral filter to the Fourier Transform removing all interferometric modulation, and (3) taking a second FFT of the filtered Fourier Transform. In their analysis they show that the precision and accuracy of $\mathrm{CO}_{2}$ total column concentrations can be improved by the application of a SBF correction to spectra in the near infrared spectral region (3800-15 $\left.750 \mathrm{~cm}^{-1}\right)$.

However, the application of a SBF correction to spectra in the mid infrared spectral region $\left(700-3800 \mathrm{~cm}^{-1}\right)$ for the analysis of many important trace gases, such as $\mathrm{O}_{3}$, is reasonable. Between $3800 \mathrm{~cm}^{-1}$ and $2000 \mathrm{~cm}^{-1}$ FTIR spectra are measured with an InSb detector. Below $2000 \mathrm{~cm}^{-1}$ FTIR spectra are usually measured with a photoconductive MCT (mercury-cadmium-telluride) detector. Spectra measured with such a MCT detector are generally considered uncorrectable (Griffith and de Haseth, 1986; Rao, 1992); during the measurement process the MCT is applied with a constant voltage which adds an unknown offset $O$ to the measured DC interferogram (Fig. 2). A preamplifier circuit diagram can be found in Fig. 6. The unknown offset perturbs the SBF correction method (Eq. 1) since the reweighting of the raw interferogram with the smoothed interferogram now amplifies the modulation by an incorrect ratio. Thus, the unknown offset has to be removed prior to a SBF correction.

Removing the unknown offset is essential to allow for a SBF correction of MCT interferograms. However, a proce-

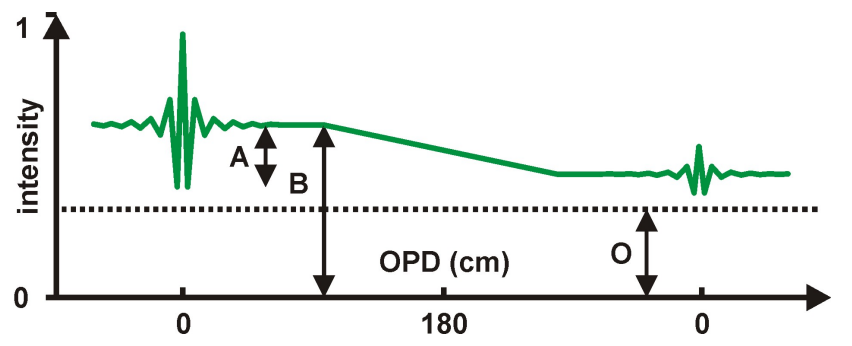

Fig. 2. Schematic of a DC interferogram measured with a MCT detector showing an unknown offset $O$. The ratio $A$ (modulation height) to $B-O$ (background intensity) demonstrates the modulation efficiency of the instrument.

dure to determine the unknown offset has not been published so far. Thus, MCT interferograms have not been corrected for SBFs in previous studies.

In Sect. 2 we present a solution to remove the unknown offset in MCT-DC interferograms allowing MCT spectra for an application of source brightness fluctuation correction. We present a method of source brightness fluctuation correction which is independent of the used detector and can, thus, be applied to spectra in the whole infrared spectral region.

In Sect. 3 we study the impact of source brightness fluctuation correction on MCT spectra. We further investigate the influence of SBF correction on the retrieval of $\mathrm{O}_{3}$ profiles and total column concentrations. In addition, we test our SBF correction method by comparing it to the method presented by Keppel-Aleks et al. (2007) based on the example of $\mathrm{CO}_{2}$ total column concentration measurements in Bialystok, Poland.

\section{Method}

The offset in MCT interferograms can be removed in two ways. In the first case, we measure the modulation efficiency

$M=\frac{A}{B-O}$

of the instrument (Fig. 2) using an InSb detector and a small optical filter (bandwidth: $1900-2700 \mathrm{~cm}^{-1}$ ). In Eq. (2) $A$ is the AC signal intensity and $B$ is the DC signal intensity plus the offset. The measurement is repeated with the MCT detector using the same filter and optical settings. Thus, we can assume that the modulation efficiency is the same for both detectors,

$M_{\mathrm{InSb}}=\frac{A_{\mathrm{InSb}}}{B_{\mathrm{InSb}}}=\frac{A_{\mathrm{MCT}}}{B_{\mathrm{MCT}}-O}$

and the offset can then be calculated as

$O=B_{\mathrm{MCT}}-\frac{A_{\mathrm{MCT}}}{M_{\mathrm{InSb}}}$ 
The variables $A_{\mathrm{MCT}}$ and $B_{\mathrm{MCT}}$, both need to be determined in the vicinity of the interferogram's centerburst to avoid errors in the offset due to a change in the source brightness. Since the offset is dependent on the modulation efficiency of the instrument, the modulation efficiency has to be determined regularly to avoid errors in the offset due to a change in the instrument alignment.

The second way to remove the offset in MCT interferograms can be applied to a pair of interferograms which is measured directly in series, if both centerbursts differ in their intensity due to SBF (Fig. 2). The modulation efficiency in each interferogram must be equal,

$M_{\mathrm{MCT}_{1}}=\frac{A_{\mathrm{MCT}_{1}}}{B_{\mathrm{MCT}_{1}}-O}=\frac{A_{\mathrm{MCT}_{2}}}{B_{\mathrm{MCT}_{2}}-O}=M_{\mathrm{MCT}_{2}}$

and the offset is calculated as

$O=\frac{A_{\mathrm{MCT}_{2}} \cdot B_{\mathrm{MCT}_{1}}-A_{\mathrm{MCT}_{1}} \cdot B_{\mathrm{MCT}_{2}}}{A_{\mathrm{MCT}_{2}}-A_{\mathrm{MCT}_{1}}}$

This method is independent of the alignment of the instrument in comparison to the first method.

For the SBF correction (Eq. 1) all operations can be performed within OPUS (version 6.5), the standard Bruker spectroscopy software. $I_{\text {raw }}$ is measured with the OPUS measurement procedure, the offset is subtracted from the raw interferogram using the OPUS calculator, $I_{\text {smooth }}$ is generated by a direct smoothing of $I_{\text {raw }}$ with the OPUS running mean function, and the reweighting is again performed by the OPUS calculator. The procedure is independent of the used detector, and it can be applied automatically and instantaneously together with the measurement process. The smoothing is accomplished by applying twice a running mean with a data point window of 1000 . The choice of the data point window is not critical as long it does not affect the modulation frequencies. Here, a reasonable lower boundary for the window size is $\mathbf{5 0 0}$ data points.

Admittedly, the OPUS running mean function still ignores datapoints at the edges of $I_{\text {raw }}$, and thus, the reweighting is inaccurate there. However, this problem is of no great consequence for the procedure; in forward-backward scans the edges of the interferogram are only of importance for the phase correction (Brault, 1987; Chase, 1982), and the phase correction can be adjusted by slightly reducing the phase resolution. In single scans the problem can be avoided by minimally reducing the resolution and phase resolution of the measurement.

In Sect. 3 DC interferograms are compared to SBFcorrected interferograms and $\mathrm{AC}$ interferograms. The $\mathrm{AC}$ interferograms are numerically deduced by subtracting the smoothed interferogram from the raw interferogram within OPUS.

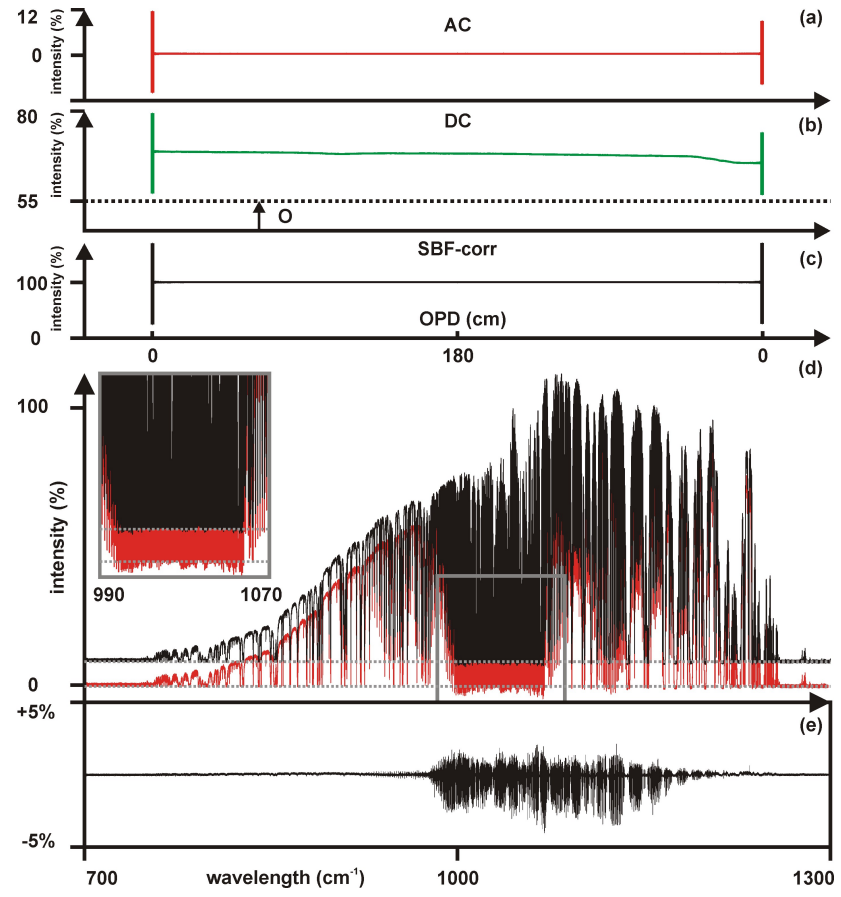

Fig. 3. SBF correction of a solar absorption MCT interferogram; (a) AC interferogram, (b) DC interferogram, (c) SBF-corrected interferogram, (d) AC spectrum and SBF-corrected spectrum (shifted along ordinate), (e) residual plot of AC spectrum and SBF-corrected spectrum.

\section{Results}

Solar absorption FTIR spectra are obtained by a Bruker IFS $120 / 5 \mathrm{M}$ and a Bruker IFS 125HR Fourier Transform spectrometer. The IFS $120 / 5 \mathrm{M}$ was applied during a field campaign aboard research vessel (RV) Sonne in 2009. Here, the problem of SBF had to be addressed in particular since the campaign was planned as a North-South transit from Japan to New Zealand crossing over the tropics, where an increased appearance of clouds was expected. By default the 120/5M measures in $\mathrm{AC}$ mode, but was adjusted to measure in DC mode with all detectors (InGaAs, Si, InSb, MCT) for the whole infrared spectral region. Measurements in the mid infrared spectral region were performed using a photoconductive MCT detector at low signal intensity in order to avoid non-linearity effects. The IFS $125 \mathrm{HR}$ is based in Bialystok, Poland, and measures in DC mode only for the near infrared range (3800-15750 $\mathrm{cm}^{-1}$ ) with an InGaAs diode and a $\mathrm{Si}$ diode detector. The spectral coverage of each individual detector used in the IFS $125 \mathrm{HR}$ and the IFS $120 / 5 \mathrm{M}$ during solar absorption measurements is shown in Table 1.

\subsection{MCT}

Figure 3 shows the SBF correction of a solar absorption FTIR interferogram measured with the $120 / 5 \mathrm{M}$ spectrometer using 
Table 1. Spectral range of different detectors used during solar absorption measurements with the 120/5M and $125 \mathrm{HR}$ instrument.

\begin{tabular}{lcccc}
\hline & MCT & InSb & InGaAs & Si \\
\hline $120 / 5 \mathrm{M}$ & $600-1500 \mathrm{~cm}^{-1}$ & $1800-4500 \mathrm{~cm}^{-1}$ & $3800-11000 \mathrm{~cm}^{-1}$ & $10000-15750 \mathrm{~cm}^{-1}$ \\
$125 \mathrm{HR}$ & - & - & $3800-11000 \mathrm{~cm}^{-1}$ & $10000-15750 \mathrm{~cm}^{-1}$ \\
\hline
\end{tabular}

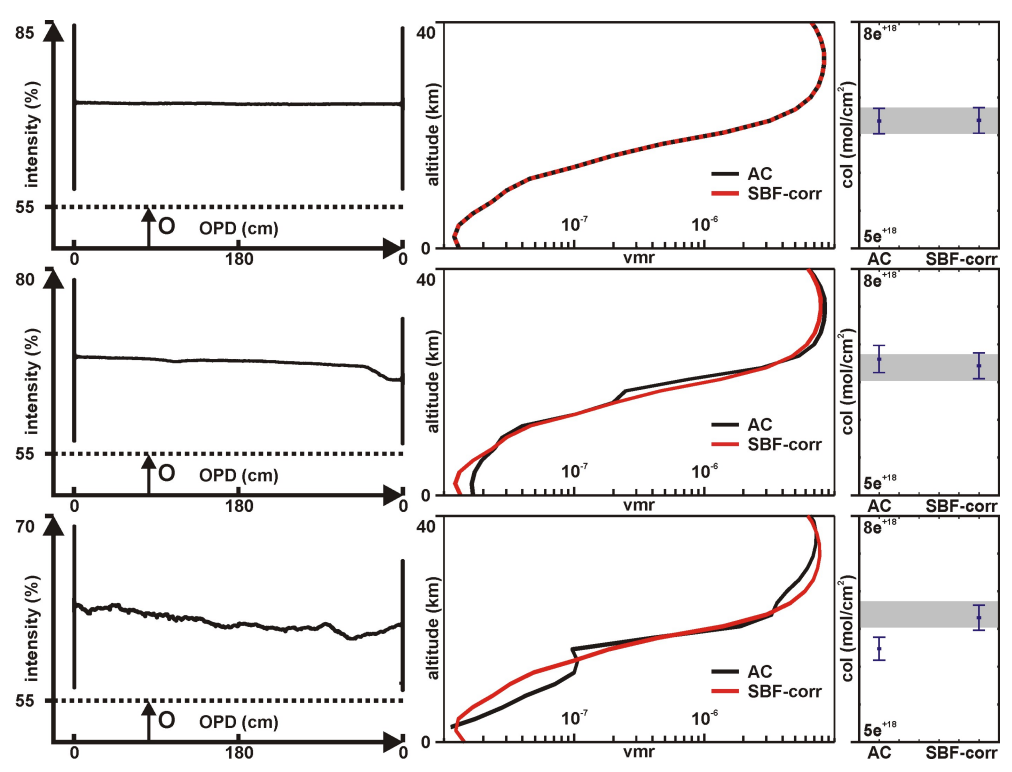

Fig. 4. Influence of SBF correction on the retrieval of $\mathrm{O}_{3}$ profiles and total column concentrations. Left: Three MCT, DC interferograms with and without the influence of SBF. Center: $\mathrm{O}_{3}$ profiles retrieved from the corresponding $\mathrm{AC}$ and SBF-corrected spectra. Right: $\mathrm{O}_{3}$ total column concentrations.

a MCT detector. In Fig. 3a-c three interferograms are shown (AC, DC, SBF-corrected), in Fig. 3d two spectra created from the $\mathrm{AC}$ and the $\mathrm{SBF}$-corrected interferograms are presented, and in Fig. 3e a residual plot determined from the AC and the SBF-corrected spectra is displayed. The interferograms' intensity is described as the percentage efficiency of the instrument's ADC (analog-to-digital converter).

The AC-forward-backward interferogram reveals that the measurement is influenced by SBF causing different heights in both interferograms (Fig. 3a). More obviously, the influence of SBF is visible in the DC interferogram (Fig. 3b). The DC interferogram indicates an intensity loss towards the end of the measurement and shows the same modulation loss as in AC mode. Furthermore, the DC interferogram features the typical MCT offset.

For the SBF correction the offset in the MCT-DC interferogram has to be removed. Following the first method (Eq. 4) the offset is calculated as $O=0.546519$ using a modulation efficiency of $M_{\mathrm{InSb}}=87 \%$ measured with the InSb detector. Following the second method (Eq. 6) the offset is calculated as $O=0.548764$ in excellent agreement to the first method (deviation of $0.41 \%$ ).

Following the removal of the offset the SBF correction with OPUS can be applied. Thereby, the phase resolution was reduced from $4 \mathrm{~cm}^{-1}$ to $4.7 \mathrm{~cm}^{-1}$ according to Sect. 2 .
The correction compensates the intensity fluctuations and reweights the interferometric modulation visible in the equalized heights in both interferograms (Fig. 3c).

The corresponding spectra of the AC interferogram and the SBF-corrected interferogram are shown in Fig. 3d. The procedure corrects spectral errors visible e.g. in the spectral range between $990 \mathrm{~cm}^{-1}$ and $1070 \mathrm{~cm}^{-1}$. Here, a variety of spectral lines are generally saturated. However, the AC spectrum shows a strong oversaturation in this range due to the incorrect expression of the line depth caused by the source brightness fluctuations. This effect is corrected in the SBFcorrected spectrum. The residual plot in Fig. 3e shows the difference between the AC spectrum and the SBF-corrected spectrum. Deviations of up to $\approx 4 \%$ are revealed.

The influence of SBF correction on the retrieval of trace gas concentrations is demonstrated in Fig. 4 based on the example of $\mathrm{O}_{3}$ retrieved from MCT-DC spectra. Three interferograms are shown which were measured in series within one hour. The first interferogram was measured under clear sky conditions, the second and third interferogram were measured under the influence of SBF (Fig. 4, left). All three interferograms show the typical MCT offset which is removed for the SBF correction using the first method with a fixed modulation efficiency of $M_{\mathrm{InSb}}=87 \%$. 
Table 2. Impact of errors in the offset determination on the retrieval of $\mathrm{O}_{3}$ total column concentrations; percentage deviations are related to the initial value $\left(6.6877 \times 10^{18} \mathrm{~mol} \mathrm{~cm}^{-2}\right)$ calculated from the undisturbed AC spectrum. Numbers are given in $10^{18} \mathrm{~mol} \mathrm{~cm}-2$.

\begin{tabular}{llllllll}
\hline Example & Offset & $O=$ Offset $O=0.54$ & $O=0.53$ & $O=0.52$ & $O=0.51$ & $O=0.50$ \\
\hline$(1)$ & 0.5455 & 6.6966 & 6.6963 & 6.6959 & 6.6955 & 6.6951 & 6.6948 \\
& & $(0.13 \%)$ & $(0.13 \%)$ & $(0.13 \%)$ & $(0.12 \%)$ & $(0.11 \%)$ & $(0.11 \%)$ \\
\hline$(2)$ & 0.5470 & 6.7150 & 6.7166 & 6.7243 & 6.7308 & 6.7363 & 6.7411 \\
& & $(0.41 \%)$ & $(0.43 \%)$ & $(0.54 \%)$ & $(0.64 \%)$ & $(0.72 \%)$ & $(0.80 \%)$ \\
\hline$(3)$ & 0.5468 & 6.6520 & 6.6234 & 6.5732 & 6.5347 & 6.5042 & 6.4795 \\
& & $(0.54 \%)$ & $(0.96 \%)$ & $(1.71 \%)$ & $(2.29 \%)$ & $(2.74 \%)$ & $(3.1 \%)$ \\
\hline
\end{tabular}

The retrieval of $\mathrm{O}_{3}$ profiles and total column concentrations was accomplished in each case for the $\mathrm{AC}$ and the SBF-corrected spectrum using the retrieval software SFIT2 (Rinsland et al., 1998). For the retrieval of $\mathrm{O}_{3}$ the standard NDACC microwindow $\left(1000-1005 \mathrm{~cm}^{-1}\right)$ was used. Since the measurements were performed within one hour, it can be assumed that the $\mathrm{O}_{3}$ concentrations have not significantly changed during this time period.

In Fig. 4 (center) three measurements of $\mathrm{O}_{3}$ profiles are shown. The first one was performed under clear sky conditions, whereas the second and the third one were performed under the influence of SBF. In the first case, the $\mathrm{O}_{3}$ profiles of the AC spectrum (AC) and the SBF-corrected spectrum (SBF-corr) are identical showing that the SBF correction has no influence on undisturbed spectra. In the second case, under the small influence of SBF the $\mathrm{O}_{3}$ profile from the AC spectrum differs from the first case. In contrast, the $\mathrm{O}_{3}$ profile from the SBF-corrected spectrum equals the undisturbed profiles in case one. In the third case, the influence of SBF is more obvious. The $\mathrm{AC}-\mathrm{O}_{3}$ profile strongly differs from the original profile. However, the $\mathrm{O}_{3}$ profile from the SBFcorrected spectrum equals the undisturbed cases.

The impact of SBF can also be seen in the $\mathrm{O}_{3}$ total column concentrations (Fig. 4, right). In the undisturbed case, the total column concentrations of the $\mathrm{AC}$ spectrum and the SBF-corrected spectrum are identical (deviation of $0.13 \%$ ). In the second and third case, the AC total column concentrations differ from the undisturbed case with a deviation of $1.74 \%$ and $6.73 \%$, respectively. The SBF-corrected total column concentrations, however, equal the undisturbed concentrations with a negligible deviation of $0.41 \%$ and $0.54 \%$, respectively.

Based on the three examples in Fig. 4 we now study the impact of errors in the determination of the offset on the retrieval of $\mathrm{O}_{3}$ total column concentrations. In Table 2 the total column concentrations of $\mathrm{O}_{3}$ (and their percentage deviations to the $\mathrm{AC}$ reference value) for the three examples and for different applied offsets are presented. In the undisturbed case (1) the offset does not affect the $\mathrm{O}_{3}$ retrieval. However, the impact of errors in the offset on the retrieval increases

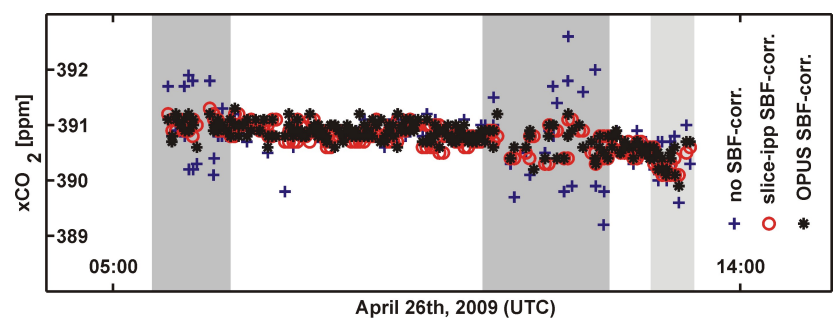

Fig. 5. Comparison of $x \mathrm{CO}_{2}$ total column concentrations from uncorrected (+), slice-ipp corrected (o), and OPUS-corrected $\left(^{*}\right)$ spectra.

in the second (2) and third (3) case. Large errors in the offset $(8.5 \%)$ lead to an error of up to $3.1 \%$ in the total column concentration. Small errors in the offset determination on the other hand do not significantly change the total column concentrations (an error of $1.3 \%$ in the offset in case (3) leads to an error of less than $1 \%$ in the total column concentration).

\subsection{InGaAs}

In Sect. 2 the source brightness fluctuation correction method used in this study to correct MCT interferograms after the removal of the offset was presented. This correction method is based on Eq. (1) which describes the reweighting of the DC interferogram following Brault (1985). Keppel-Aleks et al. (2007) used the same equation as a basis for their correction. However, the realization of the reweighting differs.

Interferogram offsets are not an issue in Keppel-Aleks et al. (2007). However, beyond the feasibility of offsetcorrection our method differs from the method in KeppelAleks et al. (2007) also with respect to the numerical implementation of the low-pass filtering. In order to prove the validity of our method, we compare our implementation to the method in Keppel-Aleks et al. (2007) using InGaAs measurements since their method is not applicable to MCT measurements.

In Keppel-Aleks et al. (2007) the smoothed interferogram $I_{\text {smooth }}$ is generated in the spectral domain within the selfmade program slice-ipp by applying a Fourier transformation 


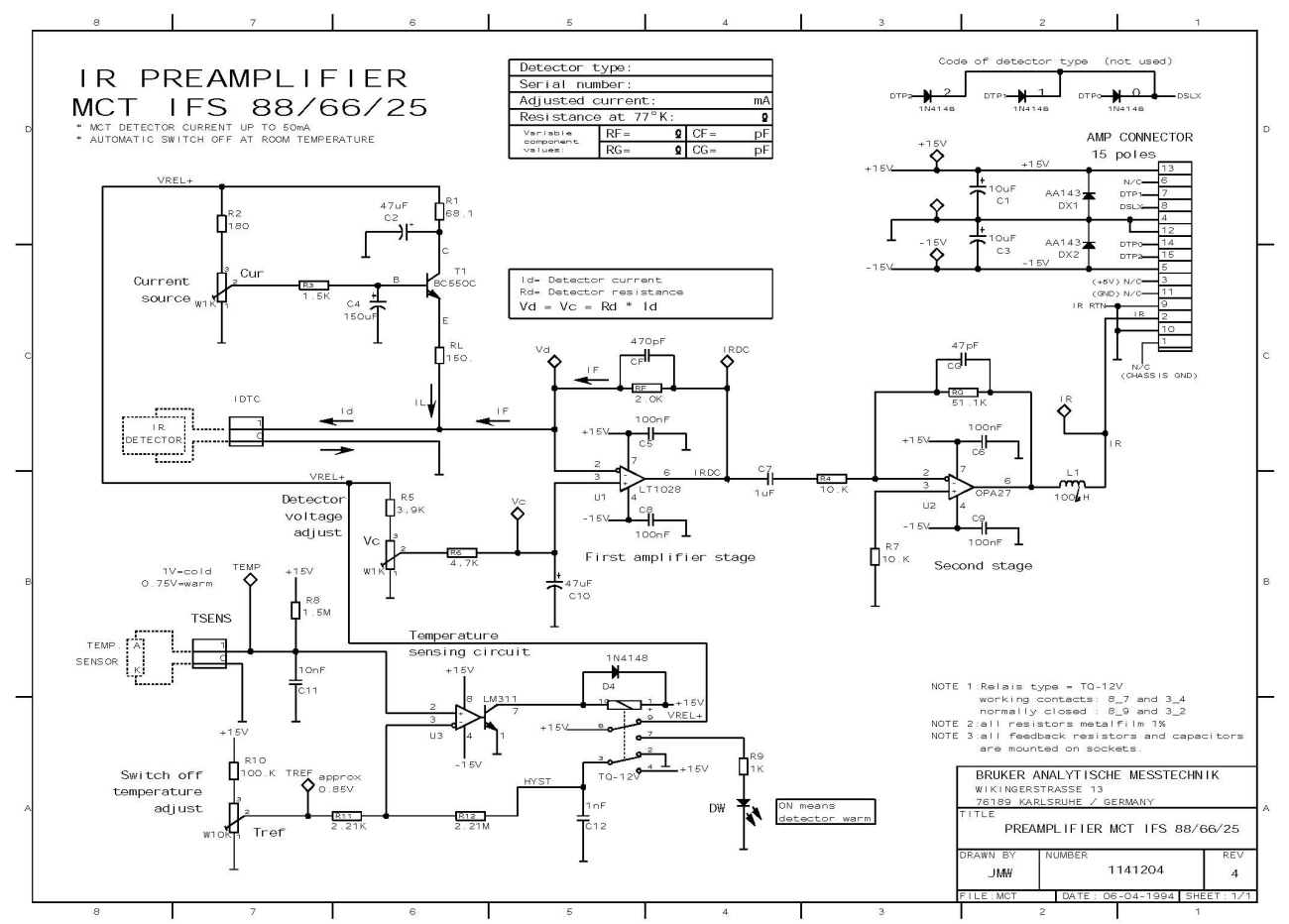

Fig. 6. Preamplifier circuit diagram of the photoconductive MCT detector (Bruker GmbH). A voltage applied to the detector element causes an unknown offset in the DC interferogram.

to the raw interferogram and cutting of the low frequencies in the corresponding spectrum. Here, the smoothing is performed in the interferogram domain by applying the standard Bruker OPUS smoothing function directly to $I_{\text {raw }}$. Using the Bruker software OPUS for the correction has two advantages compared to the method presented in Keppel-Aleks et al. (2007). First, the commercially available spectroscopic software OPUS is used which is by default applied by most of the NDACC and TCCON stations. Second, it can easily be applied to different spectral regions with different measurement parameters in contrast to slice-ipp, which is custom-tailored to the TCCON near-infrared spectral region and can not readily be used for the correction of the mid-infrared spectral region.

Due to the differences in both methods we test the method presented in this study by comparing our SBF correction method to the method presented by Keppel-Aleks et al. (2007) based on the example of $\mathrm{CO}_{2}$ total column concentration measurements in Bialystok, Poland, in April 2009. These $\mathrm{CO}_{2}$ measurements are subject to the TCCON requirements which require a high precision of $0.1 \%$. The comparison is therefore suitable for a quality test of the correction method since errors in the correction method will be reflected in the precision of the measurement.

The $\mathrm{CO}_{2}$ measurements were performed according to the TCCON standard with a $125 \mathrm{HR}$ spectrometer in single-scan mode. Likewise, the $\mathrm{CO}_{2}$ retrieval was accomplished fol- lowing the TCCON approach (microwindows: $6220 \mathrm{~cm}^{-1}$, $6339 \mathrm{~cm}^{-1}$ ) using the retrieval software GFIT (Wunch et al., 2010; Toon et al., 1992).

The comparison contains three kinds of spectra (Fig. 5): uncorrected spectra, SBF-corrected spectra according to Keppel Aleks et al. (slice-ipp SBF-corr), and SBF-corrected spectra according to the method presented here (OPUS SBFcorr). For the OPUS SBF correction the spectra were modified as discussed in Sect. 2; the resolution was reduced from $0.014 \mathrm{~cm}^{-1}$ to $0.0141 \mathrm{~cm}^{-1}$ and the phase resolution was diminished from $4 \mathrm{~cm}^{-1}$ to $4.7 \mathrm{~cm}^{-1}$. For generating $I_{\text {smooth }}$ the OPUS smoothing function is applied twice with a data point window of 1000 .

Figure 5 shows the $x \mathrm{CO}_{2}$ total column-averaged dry air mole fraction (Messerschmidt et al., 2010; Washenfelder et al., 2006) for Bialystok, Poland, in April 2009 for all three cases. All three cases agree well within the intervals where the measurements are not influenced by SBF (Fig. 5, white background). In the intervals where the spectra are influenced by SBF (Fig. 5, grey background) the precision of the $\mathrm{CO}_{2}$ total column concentrations decrease for uncorrected spectra. SBF-corrected spectra, following KeppelAleks et al. (2007), show an improvement in the precision resulting in a similar precision as for undisturbed spectra. The $\mathrm{CO}_{2}$ total column concentrations from the spectra corrected with the SBF correction method presented here show the same improvement in the precision. 


\section{Conclusions}

We showed a source brightness fluctuation correction method for solar absorption Fourier Transform infrared spectra which is independent of the detector and wavelength and can be used for a correction of the whole infrared spectral region.

We tested our source brightness fluctuation correction method by comparing it to the method used within the TCCON network (Keppel-Aleks et al., 2007) based on the example of $\mathrm{CO}_{2}$ total column concentration measurements. We found an improvement in the precision of $\mathrm{CO}_{2}$ total column concentrations identical to the TCCON approach.

Spectra in the spectral region below $2000 \mathrm{~cm}^{-1}$ measured with a MCT detector were previously considered uncorrectable due to an unknown offset in the interferogram. We presented a solution to remove the unknown offset in MCT interferograms allowing MCT spectra for an application of source brightness fluctuation correction.

We showed a source brightness fluctuation correction applied on MCT spectra which improves the quality of the spectra in terms of signal to noise ratio and spectral errors resulting in an improved retrieval of $\mathrm{O}_{3}$ profiles and total column concentrations.

Acknowledgements. We acknowledge the financial support from the Bundesministerium fuer Bildung und Forschung (BMBF) within the TRANSBROM and SO-203 projects with RV Sonne. T. Ridder is supported by the Earth System Science Research School (ESSReS).

Edited by: T. von Clarmann

\section{References}

Beer, R.: Remote Sensing by Fourier Transform Spectrometry, vol. 120, Wiley-Interscience, New York, NY, USA, 1992.

Brault, J. W.: High Resolution in Astronomy, Swiss Society of Astronomy and Astrophysics, Sauverny, Switzerland, 1985.

Brault, J. W.: High precision fourier transform spectrometry: the critical role of phase corrections, Microchim. Acta, 93, 215-227, doi:10.1007/BF01201691, 1987.

Chase, D. B.: Phase correction in FT-IR, Appl. Spectrosc., 36, 240 244, 1982.

Goldman, A., Murcray, F. J., Murcray, F. H., Murcray, D. G., and Rinsland, C. P.: Measurements of several atmospheric gases above the South Pole in December 1986 from high-resolution 3- to 4- $\mu \mathrm{m}$ solar spectra, J. Geophys. Res., 93, 7069-7074, doi:10.1029/JD093iD06p07069, 1988.

Griffith, P. R. and de Haseth, J. A.: Fourier Transform Infrared Spectrometry, vol. 83, Wiley-Interscience, Hoboken, NJ, USA, 1986.

Keppel-Aleks, G., Toon, G. C., Wennberg, P. O., and Deutscher, N. M.: Reducing the impact of source brightness fluctuations on spectra obtained by Fourier-transform spectrometry, Appl. Optics, 46, 4774-4779, 2007.
Messerschmidt, J., Macatangay, R., Notholt, J., Petri, C., Warneke, T., and Weinzierl, C.: Side by side measurements of $\mathrm{CO}_{2}$ by ground-based Fourier transform spectrometry (FTS), Tellus B, 62, 749-758, doi:10.1111/j.1600-0889.2010.00491.x, 2010.

NDACC: Network for the Detection of Atmospheric Composition Change, available at: http://www.ndsc.ncep.noaa.gov/, last access: 1 January 2011, 1991.

Notholt, J., Schütt, H., and Keens, A.: Solar absorption measurements of stratospheric $\mathrm{OH}$ in the UV with a Fourier-transform spectrometer, Appl. Optics, 36, 6076-6082, 1997.

Notholt, J., Kuang, Z., Rinsland, C. P., Toon, G. C., Rex, M., Jones, N., Albrecht, T., Deckelmann, H., Krieg, J., Weinzierl, C., Bingemer, H., Weller, R., and Schrems, O.: Enhanced upper tropical tropospheric COS: impact on the stratospheric aerosol layer, Science, 300, 307-310, doi:10.1126/science.1080320, 2003.

Rao, K. N.: Spectroscopy of the Earth's Atmosphere and Interstellar Medium, Academic Press, Boston, MA, USA, 1992.

Rinsland, C. P., Levine, J. S., Goldman, A., Sze, N. D., Ko, M. K. W., and Johnson, D. W.: Infrared measurements of $\mathrm{HF}$ and $\mathrm{HCl}$ total column abundances above Kitt Peak, 19771990: seasonal cycles, long-term increases, and comparisons with model calculations, J. Geophys. Res., 96, 15523-15540, doi:10.1029/91JD01249, 1991.

Rinsland, C. P., Jones, N. B., Connor, B. J., Logan, J. A., Pougatchev, N. S., Goldman, A., Murcray, F. J., Stephen, T. M., Pine, A. S., Zander, R., Mahieu, E., and Demoulin, P.: Northern and Southern Hemisphere ground-based infrared spectroscopic measurements of tropospheric carbon monoxide and ethane, J. Geophys. Res., 103, 28197-28217, doi:10.1029/98JD02515, 1998.

TCCON: Total Carbon Column Observing Network, available at: http://www.tccon.caltech.edu, last access: 1 January 2011, 2005.

Toon, G. C., Farmer, C. B., Schaper, P. W., Lowes, L. L., and Norton, R. H.: Composition measurements of the 1989 arctic winter stratosphere by airborne infrared solar absorption spectroscopy, J. Geophys. Res., 97, 7939-7961, 1992.

Velazco, V., Notholt, J., Warneke, T., Lawrence, M., Bremer, H., Drummond, J., Schulz, A., Krieg, J., and Schrems, O.: Latitude and altitude variability of carbon monoxide in the Atlantic detected from ship-borne Fourier transform spectrometry, model, and satellite data, J. Geophys. Res., 110, D09306, doi:10.1029/2004JD005351, 2005.

Washenfelder, R. A., Toon, G. C., Blavier, J.-F., Yang, Z., Allen, N. T., Wennberg, P. O., Vay, S. A., Matross, D. M., and Daube, B. C.: Carbon dioxide column abundances at the Wisconsin Tall Tower site, J. Geophys. Res., 111, D22305, doi:10.1029/2006JD007154, 2006.

Wunch, D., Toon, G. C., Blavier, J. L., Washenfelder, R., Notholt, J., Connor, B. J., Griffith, D. W. T., Sherlock, V., and Wennberg, P. O.: The total carbon column observing network (TCCON), Philos. T. R. Soc. A., 369, 2087-2112, 2010.

Zander, R., Stokes, G. M., and Brault, J. W.: Simultaneous detection of FC-11, FC-12 and FC-22, through 8 to 13 micrometers IR solar observations from the ground, Geophys. Res. Lett., 10, 521-524, doi:10.1029/GL010i007p00521, 1983. 